\title{
Progress towards development and adoption of integrated management systems against flystrike and lice in sheep
}

\author{
D.J.W. COLE and A.C.G. HEATH \\ AgResearch, Wallaceville Animal Research Centre, PO Box 40063, Upper Hutt, New Zealand
}

\begin{abstract}
Ectoparasites of sheep, principally blowflies and lice, cost the New Zealand farming industry an estimated $\$ 60$ million each year. This figure includes the cost of chemicals and labour used to treat or prevent flystrike and lice, together with the lost production. An Integrated Pest Management (IPM) trial project involving four groups of farmers (overall total 15) in four regions of New Zealand has been set up with the aim of adopting a holistic approach to managing these parasites and already early benefits have accrued to the farmers involved. Procedures were set up to reduce insecticide residues in wool by using techniques such as jetting which apply less chemical to the sheep but target it more appropriately and maintain or improve protection against flystrike and control of lice. As a consequence, savings in chemical and labour costs have been made. On two farms in particular, savings on these items ranged from $\$ 578$ (27\% reduction) on a property with 2000 Corriedale ewes to $\$ 3608$ (52\% reduction) on the other property with 8000 Romney ewes. An understanding that clean, healthy sheep, free of nematodes and dags, are less susceptible to flystrike and lice has also assisted in controlling the diseases. This paper also discusses the potential for biological control against blowfly populations, with large fly traps, sterilising baits and parasitoid wasps all being investigated. Research continues on specialist pastures such as birdsfoot trefoil (Lotus corniculatus) and sulla (Hedysarum coronarium) to evaluate the practicalities of incorporating their use in management systems for both nematode and flystrike control.
\end{abstract}

Keywords: biological control, blowflies, flystrike, insecticides, IPM, lice, pasture varieties, sheep

\section{Introduction}

Integrated management is a means of reducing "chemical" input to farming systems. It has been seen as a "last resort" in advanced agricultural systems (Horn 1988) and a reflection of increasing difficulty in maintaining production through, in the case of parasites, almost total reliance on insecticides and anthelmintics.
There is also a general change in the attitude of society to the use of "chemicals" in farming and increasing pressure to modify this use.

The concept of integrated management is also an excellent method for encouraging farmers to think of farming as applied ecology and an interplay of interrelated systems where the use of chemicals is only one of the variables. This requires a knowledge of the bionomics of the parasites under consideration together with ways in which a variety of population control mechanisms can be employed within the framework of the ecological knowledge. To make use of this information, farmers also need to know how to monitor and recognise changes in parasite numbers and activity.

The extent to which all obvious facets of farm management (husbandry, good stock maintenance, adequate nutrition) can affect the parasites needs to be understood together with the less obvious facets such as biological "friends" (predators and parasites) and the identification of breeding or resting sites for facultative parasites. Other means of disrupting parasite behaviour or modifying their effects are explained and practical ways of introducing them into the farm system are suggested.

In New Zealand, farmers are faced by the productionlimiting problems of flystrike and lice in their sheep and for a number of years we have been working on the development and implementation of technologies and systems for the integrated management of these two parasites. Heath (1998) described the principles of this systems approach to the control of ectoparasites of sheep.

Blowfly strike is one of the major animal health problems faced by sheep farmers in New Zealand. Total cost of this disease is estimated to be $\$ 40 \mathrm{M}$ annually, made up of lost production, productivity, deaths, and costs of chemical applications. Add another $\$ 20 \mathrm{M}$ for the effects of lice through downgrading of pelts affected with the louse-related defect, cockle, and from poor quality wool, particularly in fine wool sheep. There is also the considerable worry and trouble farmers face when agonising over how to deal with these pests. An even more important consideration for the farmer is one of animal welfare. Flystrike is a particularly nasty disease and sheep left untreated can suffer a slow and painful death.

Although this paper is principally about flystrike, lice have been controlled traditionally by the same 
insecticides so both must be considered together when formulating an IPM strategy. Herein lies a problem. Not only are these parasites becoming resistant to some of the insecticides thus limiting the range of effective products available, but concerns about the effect of chemicals on the environment may further limit their use. Other, more acceptable methods of control must be found which reduce reliance on traditional insecticides and perhaps eventually replace them altogether.

\section{The Integrated Pest Management (IPM) farm trials}

The AgResearch Wallaceville, Entomology Group's flystrike and louse IPM trial project has been running for 5 years, the last two in collaboration with WoolPro and more recently the Southern North Island Sheep Council. The project is a pragmatic response to the continued pressure on New Zealand wool-growers to reduce insecticide levels in exported wool to standards that conventional saturation dipping does little to help. If European Union demands for stringent Environmental Quality Standards cannot be met, wool markets are threatened. A network of farms is involved in the programme - five in Canterbury, five in Wairarapa, four in Waikato/King Country, and one in Wairoa selected to cover a range of climatic conditions, breeds of sheep and management practices. As a preliminary, the knowledge base of farmers in the scheme was broadened through access to appropriate extension material and field days, as well as one to one discussions and instruction. In addition, monitoring systems were established to enable farmers to have an enhanced rational base for decision making.

\section{Monitoring}

Systems were set up to monitor fly numbers and activity by trapping and collection of maggots from struck sheep, and farmers kept a diary of flystrike prevalence throughout the year. Flies and maggots were identified in the laboratory and provided a picture of flystrike epidemiology on each farm as well as establishing a baseline for measuring changes related to the impact of the new control strategies. Farmers were shown how to detect lice and measure the severity of infestations. A system of wool sample collection has been established so that insecticide residues can be measured and progress charted towards reduced residues, a measure of the success of low-chemical input farming.

\section{Development of strategies}

Using individual farm information such as topography, climate and management practices, alternative IPM strategies are considered and, if appropriate, one is implemented and assessed for efficacy. A crucial component of the project is the links the farmers have through discussion groups, Meat NZ/WoolPro Monitor Farm programme community groups, veterinarian practices and the WoolPro Extension Specialist networks. These forums are used to communicate and demonstrate to other farmers the practical benefits of IPM.

\section{Components of the integrated system}

An integrated system is a mixture of conventional animal husbandry and dipping practices, together with the addition of biological control components. The conventional aspects may require some modification by the farmer and the biological aspects may need some explanation. Once rational animal husbandry and dip use systems are in place, other components of the integrated system are introduced gradually or at a pace and in a sequence that suits the individual farmer. This is an evolutionary approach to changing farm management and is to be recommended over any prescriptive method.

\section{Husbandry components of IPM}

Employing the sound principles and practices of sheep husbandry can go a long way to countering the effects of blowflies and lice. A clean sheep is a healthy sheep or a healthy sheep is a clean sheep? Which comes first? In the case of ectoparasites, both apply. Clean sheep tend to be less attractive to flies and well-fed sheep tend to be less prone to lice. Even though these desirable states are not completely fool-proof, they are nevertheless an excellent place to start for a farmer who is interested in integrated management.

Dags, whether pasture or nematode-induced attract flies (although sheep do get flystruck on non-daggy parts of the body), as does footrot and other microbiological conditions such as dermatophilosis. Dags need not be just removed, but can be partly prevented by docking tails to the recommended length, i.e., to the tip of the vulva in ewes (Anon 1996). An over-short tail prevents the sheep from lifting the perianal wool away from the anus during defaecation. Urine stain (scald) is also a major source of flystrike risk. A damaged vulva or one malformed due to a genetic abnormality can direct urine sideways onto leg wool. Selection within flocks may eliminate this trait if it is genetically based.

However, sheep can have dags for other reasons, for example when hyper-immune to nematodes or when put onto lush fresh pasture, so it may be necessary shear off dags if they cannot be prevented. Footrot and other 
infections also predispose to flystrike. Strategic timing of shearing should also be considered. Sheep with short wool at any time during the fly season are generally less likely to get struck, but if at risk, can be treated with insecticides more effectively. Dip residues are then likely to be lower at the next shearing than if sheep were dipped with long wool.

\section{Chemical components of IPM}

In most farming businesses at present, it would be impractical to dispense completely with chemical dips, so the prime objective is to use these as effectively as possible, i.e., to use the least possible amount of the most appropriate chemical to give the greatest effect at the lowest cost, resulting in the minimum residue in the wool at the next shearing.

Traditionally, insecticides have been applied to groups of sheep using whole-animal saturation methods. We emphasise to farmers that apart from feeding, which is carried out on a flock basis, all other animal manipulations are done individually, so there is no reason why dipping cannot fall into the same category.

In this way, we emphasise that care is an important component of dip application and it also provides an opportunity for applying the dip exactly where it does the most good; along the back and around the breech in the case of flystrike prevention. This immediately reduces applied chemical volume by about at least $75 \%$, and by $100 \%$, the amount of residual dip wash that must be disposed of to the detriment of the environment.

Louse treatments are best carried out off-shears, regardless of what time of year this occurs. Treatment immediately after shearing offers a window of opportunity when louse numbers have been reduced by physical removal with the fleece, and the dip chemical can more easily penetrate to skin level where lice live.

\section{IPM dipping strategies}

The initial focus of our IPM project and its implementation was to ensure each farmer in the study groups was using dip chemicals in the most effective way. There were several aspects to consider. Was it necessary to treat with a chemical at all? Was the chemical being considered the most appropriate one for the purpose? Was the chemical being applied at the most appropriate time? Was the chemical being applied by the most effective method? Was the least possible amount of chemical being applied to obtain the desired control or period of protection? Was the final choice of treatment leading to an acceptable level of chemical residue in the wool at the next shearing?

For farmers to consider a change to a different treatment regime, there is often a need to demonstrate more than just reduced chemical residues in the fleece. At present, particularly in a wool market of depressed prices, there is no obvious and immediate financial incentive for many farmers to reduce residues, although those in quality programmes may have markets restricted if they do not comply with the requirements. However, we have been able to demonstrate clear and significant benefits in terms of improved performance of chemicals and reductions in cost of both chemicals and their application.

Two examples are given which are representative of savings made by many farmers in our trial groups (Table 1).

Table 1 Comparison of annual ectoparasite control costs (\$ chemical + labour) on two farms before and after introduction of IPM strategies.

\begin{tabular}{lcc}
\hline & Farm A (NI) & Farm B (SI) \\
\hline Pre-IPM & 6892 & 2104 \\
IPM 1 & 4649 & 1526 \\
IPM 2 & 3284 & \\
Saving & 3608 & 578 \\
(\% reduction) & $(57)$ & $(27)$ \\
\hline
\end{tabular}

Farm A is a 1400 ha North Island hill country property, running 8000 Romney ewes. Pre-IPM ectoparasite control strategy was: a treatment for lambs at docking with an insect growth regulator (IGR) for fly; handjet lambs at weaning (November) with an organophosphate (OP) for fly; shower dip all sheep at least once over summer with an OP for fly and lice - all ewes repeat treatment (hand-jetted) for fly in March. After the introduction of IPM (IPM 1) the strategy was: docking treatment as before; handjet lambs (January after shearing) with an IGR and repeat in April; handjet all other sheep once over summer for fly; use synthetic pyrethroid (SP) backline treatment off-shears on all breeding stock for lice. A further 2 years into the project (IPM 2), it was established that lice were no longer present in the flock so the SP backline treatment was discontinued.

Farm B is a 300 ha South Island property, running 2000 Corriedale ewes. The pre-IPM ectoparasite control strategy was: all sheep plunge dipped with OP in January for fly and lice. After the introduction of IPM (IPM 1) the strategy was: all sheep hand-jetted December/January for fly with IGR; backline SP treatment for ewes and hoggets off-shears for lice.

In addition to the significant cost savings, other benefits accrued. With the change to hand-jetting there was no waste dip wash to dispose of, and the change from mammalian-toxic OPs to non-toxic IGRs greatly improved operator safety. Although we were not able to 
obtain pre-IPM wool residue levels for either of these two farms in Table 1, residue data from other farms in a WoolPro survey (S. Edwards pers. comm.) indicated that the change from shower or plunge dipping to handjetting is likely to have substantially reduced the residue levels.

\section{Biological components of IPM}

\section{Environmental knowledge}

Utilising knowledge of the biology of blowflies can also help reduce risk of flystrike. Strike flies need sources of sugar, protein and water to survive and breed. On a farm these are generally found in areas of bush or scrub, often in gullies where carrion may be overlooked. Gullies also provide shelter for flies which can't fly in excessively windy conditions. Where possible, at times when sheep are likely to be susceptible to flystrike, they should be grazed in open country and, if possible, at lower than usual stocking rates.

\section{Large-scale fly-trapping and behavioural disruption} Working on the assumption that reducing the populations of strike-causing blowflies will in turn reduce the prevalence of flystrike in sheep, a number of attempts have and are being made to quantify the effects of this approach, both here and in Australia (Anderson et al. 1990; Atkinson \& Leathwick 1995; Dymock \& Forgie 1995). Whilst these and other studies have shown that fly numbers can be significantly reduced using intensive trapping, the resultant effects on flystrike are somewhat equivocal, though our work (unpublished data) showed flystrike was reduced by an average of $30 \%$ in a carefully controlled trial on multiple farmlets.

However, we are encouraged sufficiently to promote the use of large fly traps baited with offal, and placed at strategic points round farms, either in areas where flies breed and congregate or adjacent to flocks of susceptible sheep. Traps can be used either at the onset of fly activity with the intent of depleting breeding populations, or at any time to disrupt the behaviour of flies by acting as surrogate sheep, mimicking the principle of disruption by pheromone technology commonly employed with crop and fruit pests.

Many farmers in our IPM programme are using traps made from $10 \mathrm{~L}$ buckets and are reporting large catches of flies. A Farmer Initiated Technology Transfer (FITT) trial in the Awatere Valley in Marlborough using these bucket traps is now into a second year and is showing promising results in at least reducing strike-fly populations on farms where the traps are sited (P. Anderson pers. comm.). Several similar trap designs are now commercially available in New Zealand, but although the marketers are claiming success in flystrike prevention, no evidence has been produced thus far to substantiate these claims.

In the UK another technique tested was the use of target traps, baited with liver and sodium sulphide, and coated with sugar and an IGR (triflumuron) mixture (Smith \& Wall 1998). The sugar/triflumuron is ingested when flies are attracted to the targets and the triflumuron renders infertile any eggs subsequently laid by female flies. This work demonstrated a dramatic reduction in fly numbers on properties using the target traps. Some commercially sponsored preliminary work on assessing the potential of this technique has begun in New Zealand.

\section{Disposal of sheep and other animal carcasses}

Animal carcasses provide a breeding habitat for many insect species including strike flies (Dymock \& Forgie 1993). The number of flies produced from a carcass is roughly proportional to its weight. A small carcass such as a mouse can produce over 500 flies, and a small/ medium sized carcase such as a possum can produce around 3000 (Heath \& Appleton unpublished data) whereas sheep carcasses have been shown to produce between 18000 and 63000 flies in Australia (Anderson et al. 1988). There is a dilemma here, whilst fly larvae and other insects perform a useful function in breaking down carcasses, it is not desirable to have the next generation of adult flies liberated into the environment where their next host may be a live sheep. Therefore disposal of carcasses is desirable (and a legal obligation where sheep are concerned) though in many situations impracticable, particularly when considering small carrion.

\section{Parasitoid wasps}

Several parasitoid wasp species that parasitise fly larvae or pupae are either endemic or have been introduced into New Zealand. Investigations have been undertaken to determine their geographical distribution and assess their potential for control of many blowfly species (Bishop 1998). Preliminary studies have shown that blowflies emerging from carrion can be reduced in number by up to $50 \%$ due to depredation by parasitoids (D.M. Bishop pers. comm.).

Further work will be necessary to assess whether parasitoids can be manipulated to influence the prevalence of flystrike and if it is both viable and practical to either introduce wasp species in areas where they are not currently present or to artificially enhance their numbers in areas where they are present.

\section{Pasture utilisation}

Faecal accumulation (dags) around the breech is one of the major predisposing factors for flystrike (Miller 1939). 
Grazing sheep on pasture species that reduce dags or the moisture content of faeces has already been shown to assist in reducing flystrike prevalence (Leathwick \& Atkinson 1995, 1998). The effects appear quite pronounced in forages containing condensed tannins such as birdsfoot trefoil (Lotus corniculatus) and sulla (Hedysarum coronarium) (Niezen et al. 1998). To gain maximum benefit from the positive effects of these pastures, careful consideration will need to be given as to how they can be incorporated into normal grazing management to complement ectoparasite IPM.

It is frequently stated that good quality pasture is the best medicine farmers can give their stock, but skilful pasture management is not easily achieved given the interplay between market fluctuations and the relative unpredictability of the weather. However, there is still the potential to use pasture as another weapon in the anti-ectoparasite armoury.

\section{Conclusions}

The advantage of IPM is that it has built-in flexibility, and will allow internal change, although not necessarily the same outputs. This appears to be a price that has to be paid for low-chemical-input farming, but if the philosophy of sustainability of resources is to have any validity then integrated management can be applied universally in all farming systems.

The benefits will not necessarily be consistent year by year as flystrike has an annual variation which is climate driven and as such is independent of IPM suppressive effects which are of local influence only. As flystrike prevalence varies, so can the individual components of the IPM programme vary, and in years of high "fly pressure" more dip or more labour may be needed. Furthermore, louse populations can be influenced by, for example, shearing patterns and feed availability which in turn are partly climate driven.

We have discussed how sound sheep husbandry combined with strategic and better targeted use of available insecticides should improve control of flystrike and lice, and save farmers a significant amount of money. We have also identified that the use of biological control methods and of a number of pasture species have potential to be incorporated into the IPM system with further economic benefits as well as reducing reliance on traditional chemicals.

\section{ACKNOWLEDGEMENTS}

The financial support of WoolPro and the Southern North Island Sheep Council is gratefully acknowledged, as is the co-operation of the WoolPro Extension Specialists and the farmers in the IPM trials. The helpful comments of two anonymous referees are also appreciated.

\section{REFERENCES}

Anderson, J.M.E.; McLeod, L.J.; Shipp, E.; Swan, A.; Kennedy, J.P. 1990. Trapping sheep blowflies using bait-bins. Australian veterinary journal 67: 93-97.

Anderson, P.J.; Shipp, E.; Anderson J.M.E.; Dobbie, W. 1988. Population maintenance of Lucilia cuprina (Weidemann) in the arid zone. Australian journal of zoology 36: 241-249.

Anon 1996. Code of recommendations and minimum standards for the welfare of sheep: Code of animal welfare No. 3. Animal Welfare Advisory Committee. p. 17.

Atkinson, D.S.; Leathwick, D.M. 1995. Evaluation of large scale trapping of flies as a means of reducing the incidence of flystrike in lambs. Proceedings of the New Zealand Society of Animal Production 55: 193-195.

Bishop, D.M. 1998. Parasitic Hymenoptera reared from dung-breeding Diptera in New Zealand. New Zealand entomologist 21: 99-106.

Dymock, J.J.; Forgie, S.A. 1993. Habitat preferences and carcase colonisation by sheep blowflies in the northern North island of new Zealand. Medical and veterinary entomology 7: 155-160.

Dymock, J.J.; Forgie, S.A. 1995. Large-scale trapping of sheep blowflies in the northern North Island of New Zealand using insecticide-free traps. Australian journal of experimental agriculture 35: 699-704.

Heath, A.C.G. 1998. IPM: An ectoparasite management tool for farmers. Proceedings of the 28th Seminar, the Society of Sheep and Beef Cattle Veterinarians, NZVA, Dunedin. Publication No. 180, Continuing Education, Massey University, Palmerston North. pp. $25-36$.

Horn, D.J. 1988. Ecological approach to pest management. New York, The Guilford Press.

Leathwick, D.M.; Atkinson, D.S. 1995. Dagginess and flystrike in lambs grazed on Lotus corniculatus or ryegrass. Proceedings of the New Zealand Society of Animal Production 55: 196-198.

Leathwick, D.M.; Atkinson, D.S. 1998. Influence of different proportions of Lotus corniculatus in the diet of lambs on dags, flystrike and animal performance. Wool technology and sheep breeding 46: 353-359.

Miller, D. 1939. Blow-flies (Calliphoridae), and their associates in New Zealand. Cawthron Institute Monographs: No. 2, 75 p.

Niezen, J.H.; Robertson, H.A.; Waghorn, G.C.; Charleston, W.A.G. 1998. Production, faecal egg 
counts and worm burdens of ewe lambs which grazed six contrasting forages. Veterinary parasitology 80 : $15-27$.

Smith, K.E.; Wall, R. 1998. Suppression of the blowfly Lucilia sericata using odour-baited triflumuronimpregnated targets. Medical and veterinary entomology 12: 430-437.

Waghorn, G.C.; Gregory, N.; Todd, S.; Wesselink, R. 1999. Dags in sheep; a look at faeces and reasons for dag formation. Proceedings of the New Zealand Grassland Association 61: 43-49. 